\title{
Conocimiento, uso y valor cultural de seis presas del jaguar (Panthera onca) y su relación con éste, en San Nicolás de los Montes, San Luis Potosí, Mexico
}

\section{Knowledge, use and cultural value of six prey of jaguar (Panthera onca) and their relationship with this species in San Nicolás de los Montes, San Luis Potosí, Mexico}

\author{
Dulce M. Ávila-Nájera1 ${ }^{1}$, Octavio C. Rosas-Rosas², Luis A. Tarango-Arámbula²*, Juan F. Martínez-Montoya² \\ y Enrique Santoyo-Brito ${ }^{1}$ \\ ${ }^{\text {I} C o l e g i o ~ d e ~ P o s t g r a d u a d o s, ~ C a m p u s ~ M o n t e c i l l o . ~ C a r r e t e r a ~ M e ́ x i c o-T e x c o c o, ~ K m . ~ 36.5, ~ M o n t e c i l l o, ~} 56230$ Texcoco, Estado de México, México. \\ ${ }^{2}$ Colegio de Postgraduados, Campus San Luis Potosí. Iturbide 73, 78600 Salinas de Hidalgo, San Luis Potosí, México. \\ *Correspondencia: ltarango@colpos.mx
}

\begin{abstract}
Resumen. Durante 2007 se obtuvo información del conocimiento y la percepción que los pobladores del ejido tienen sobre el jaguar, sus presas y los factores que afectan la distribución y su conservación. Mediante el índice de importancia cultural (IIC) se determinó el conocimiento que los ejidatarios tienen de las presas, y el uso y valor que les otorgan. Las presas que reconocieron importantes fueron venado cola blanca (Odocoileus virginianus), temazate (Mazama temama), pecarí (Tayassu pecari), tejón (Nasua narica), armadillo (Dasypus novemcinctus) y tepezcuintle (Cuniculus paca); $72 \%$ de los encuestados también identificaron especies domésticas como presas del jaguar. Según su percepción, las más abundantes son pecarí, tejón y armadillo. El valor de uso más importante es el alimenticio, puesto que $82 \%$ de los encuestados ha consumido alguna especie silvestre. El valor más alto del IIC fue para el venado (27.17). En el ejido se considera perjudicial el jaguar por alimentarse del ganado. Los pobladores y los jaguares utilizan las mismas especies para obtener beneficio. El presente estudio constituye una base para el desarrollo de trabajos tendientes a manejar y conservar los recursos naturales que existen en el ejido considerando las necesidades de la población humana, de su fauna silvestre y la relación entre éstas.
\end{abstract}

Palabras clave: pobladores rurales, manejo, recursos, índice de importancia cultural.

\begin{abstract}
During 2007, we obtained information about the knowledge, perception of rural people regarding jaguar, its preys and the factors that affect its distribution and conservation. Additionally, we determined with the Index of Cultural Importance the knowledge, use and value that rural people assign to preys of the jaguar. They recognized as important prey species of the jaguar to white-tailed deer (Odocoileus virginianus), brocket (Mazama temama), pecari (Tayassu peccari), coati (Nasua narica), armadillo (Dasypus novemcinctus) and paca (Cuniculus paca). Most of the villagers (72\%) identified also domestic species as preys of the jaguar. According to villager's perception, the more abundant species were pecari, coati and armadillo. Wildlife use for food was the most important since $82 \%$ of the interviewed people have consumed some species. The highest value of Cultural Importance was for white tailed deer (27.17). Jaguars in the ejido are considered harmful animals since they prey on livestock. Rural people and jaguars benefit from same wildlife species. The present study is the framework to develop future projects focused on managing and conserving natural resources that exist in the ejido San Nicolás de los Montes, San Luis Potosí.
\end{abstract}

Key words: rural people, management, resources, Index of Cultural Importance.

\section{Introducción}

México es un país megadiverso debido a su ubicación geográfica, compleja historia geológica, heterogeneidad ambiental, topografía accidentada y su localización entre 2 regiones zoogeográficas, la neártica y la neotropical (Toledo, 1988; Ceballos y Oliva, 2005). En cuanto a riqueza se refiere, ocupa el tercer lugar en el mundo

Recibido: 30 septiembre 2009; aceptado: 15 marzo 2011
(Ramamoorthy et al., 1998). Específicamente se han registrado 475 mamíferos terrestres que se distribuyen en 12 órdenes, 34 familias y 165 géneros (Ceballos et al., 2002) y representan aproximadamente el $10 \%$ del total mundial de las especies de mamíferos que se conocen (Challenger, 1998).

En México, varios han sido los intentos y esquemas para manejar los recursos naturales; entre otros, colaboración internacional para la vida silvestre y biodiversidad, Áreas Naturales Protegidas (ANP), creación de dependencias administrativas federales, promulgación de leyes, Regiones 
Terrestres Prioritarias de Conservación de la Biodiversidad (RTP), Unidades de Manejo para la Conservación de Vida Silvestre (UMAS) y regulación de caza (Valdez et al., 2009). Sin embargo, para lograr el objetivo de manejo se deben contemplar prácticas basadas en un modelo que provea bienes y servicios a una creciente población humana sin poner en riesgo los recursos naturales (Bezaury-Creel y Gutiérrez-Carbonell, 2009). Para ello, la sociedad mexicana debe valorar la biodiversidad y considerar sus contribuciones en materia, ambiental, social, cultural y económica; asimismo, debe facilitar la toma de decisiones relacionadas con su conservación y uso sustentable mediante la diversificación productiva en beneficio de las comunidades locales (CONABIO, 2000).

El ejido San Nicolás de los Montes (SNM) en San Luis Potosí cuenta con recursos faunísticos sobresalientes, como venado cola blanca (Odocoileus virginianus), venado temazate (Mazama temama), pecarí de collar (Pecari tajacu), puma (Puma concolor), jaguar (Panthera onca), ocelote (Leopardus pardalis), tigrillo (L. wiedii), mapache (Procyon lotor) tepezcuintle (Cuniculus paca) y armadillo (Dasypus novemcinctus), entre otros. Algunas de estas especies constituyen presas del jaguar (Núñez et al., 2000; Hoogesteijn et al., 2006; Rosas-Rosas et al., 2008), $\mathrm{y}$ en virtud de que los pobladores las utilizan con diversos fines, frecuentemente enfrentan una presión de caza alta. No obstante, el conocimiento que existe en México sobre su uso y valor cultural es escaso (Aranda et al., 1999; Monrroy-Vilchis et al., 2008).

Los bosques tropicales constituyen una fuente de recursos para los pobladores locales, siendo la fauna silvestre un componente importante con la que el hombre mantiene una estrecha relación (Barbarán, 2004; LópezCabrera et al., 2005). Los seres humanos han adquirido conocimientos sobre ésta, la utilizan y le han otorgado valores (Aldana et al., 2006), como los mágico-religiosos, ecológicos, genéticos, alimenticios, comerciales, cinegéticos, turísticos, recreacionales, científicos (Ojasti, 2000) y culturales.

Purdy y Decker (1989) definen el valor cultural como la percepción y grado de conocimiento empírico o tradicional que el hombre tiene de los recursos naturales. Por ello, es necesario entender el uso que los lugareños del ejido San Nicolás de los Montes (SNM) hacen de la fauna silvestre y cómo se afecta el número de presas, el comportamiento y procuración de recursos por el jaguar.

La fauna silvestre forma parte de las costumbres tradicionales de los pobladores rurales y hasta ahora sólo se han realizado trabajos para describir sus usos y valores. Turner (1988) plantea la necesidad de establecer el nivel de importancia de las especies (plantas) de una manera más objetiva y evalúa el significado cultural de éstas basándose en una puntuación, la cual se obtiene de la asignación de valores a los factores que contribuyen a su significancia. Los resultados combinados producen un índice de importancia cultural (IIC) para una especie en particular. En el presente estudio se consideró pertinente utilizar en animales el índice cultural propuesto por Turner.

El trabajo tuvo 2 propósitos: 1), obtener información sobre el conocimiento y percepción que tienen los habitantes del ejido San Nicolás de los Montes, San Luis Potosí sobre el jaguar, sus presas y los factores que afectan la distribución y conservación de éstas y 2), mediante la aplicación del IIC, determinar el conocimiento, el uso y valor que otorgan los ejidatarios a las presas del jaguar. Adicionalmente, se discute acerca de las necesidades que tienen los pobladores de un complemento alimenticio, de la abundancia de presas del jaguar en la zona, de las necesidades de esta especie en términos de alimento y espacio, y de las especies domésticas y su manejo.

Esta investigación forma parte de un estudio integral que realiza el Colegio de Postgraduados sobre el conocimiento del jaguar en el estado de San Luis Potosí, que en su primera fase definió la presencia de este felino en la región (Villordo, 2009).

\section{Materiales y métodos}

El ejido de San Nicolás de los Montes contaba en 2005 con 367 personas (INEGI, 2008). Se ubica en el municipio de Tamasopo, en la Huasteca potosina, entre los $99^{\circ} 25^{\prime}$ 30.34 ' O y $22^{\circ} 7$ ' $11.65^{\prime} \mathrm{N}$ y a una altitud que va de los $360 \mathrm{a}$ los $860 \mathrm{~m}$. El ejido forma parte de la sierra Madre Oriental; la temperatura mínima es de $11.4^{\circ} \mathrm{C}$ en el mes de enero y máxima de $34.5^{\circ} \mathrm{C}$ en mayo; la precipitación anual fluctúa entre 23.7 y $365.7 \mathrm{~mm}$; el clima es semicálido húmedo con lluvias en verano. En él confluyen ríos permanentes e intermitentes que desembocan en el río Gallinas (INEGI, 2003). La vegetación predominante es bosque de encino (Quercus oleoides) y bosque tropical subperennifolio (Puig, 1991). Las principales actividades económicas son la agricultura y la ganadería (INEGI, 2003).

Para obtener información sobre el conocimiento y percepción que tienen los ejidatarios sobre el jaguar, sus presas y los factores que afectan la distribución y conservación de éstas, durante mayo-agosto 2007 se realizó una encuesta utilizando un diseño de muestreo aleatorio simple sin reemplazo y sin discriminar edad $(\geq$ 17 años) o sexo; el tamaño de muestra se calculó con el uso de la fórmula:

$$
n=\frac{N p q}{(N-1) D p q}
$$


donde: $n=$ tamaño de muestra a encuestar; $N=$ tamaño poblacional; $p=$ proporción; $q=1-p ; D=\mathrm{B} / 4$ (donde $\mathrm{B}=$ límite de los pobladores error de estimación).

Para conocer el uso que los pobladores dan a las especies de fauna en la zona y el valor cultural que se les otorga, en particular a las presas del jaguar, se realizó una primera encuesta en la que se identificaron dichas presas (aquellas especies que recibieron más menciones en la encuesta), las cuales fueron O. virginianus, M. temama, P. tajacu, N. narica, C. paca y D. novemcinctus. Con base en esta información se realizaron otras encuestas durante el mes de octubre de 2007, para las cuales se utilizó el mismo diseño de muestreo y fueron de respuesta cerrada. Los resultados de esta fase se cuantificaron con base en el índice de importancia cultural (IIC) propuesto por Turner (1988), quien en su metodología otorga valores predefinidos para cada uso de las especies, y modificado posteriormente por Figueroa-Solano (2000), quien no otorga ningún valor a los usos ni distingue entre la parte usada, como lo hace Turner

$$
I I C_{z}=\frac{\Sigma\left(l u_{z}+f m_{z}+V u t_{z}\right)}{300}
$$

donde:

$$
\begin{gathered}
I u_{z}=\frac{\text { Núm. de usos de la sp. } x}{\text { Núm. total de usos para todas las ssp. }} * 100 \\
F m_{z}=\frac{\text { Núm. de menciones de la sp. X para todos los usos }}{\text { Núm. total de menciones de todas las spp. para todos los usos }} * 100 \\
V u_{z}=\frac{\text { Núm. total de menciones de la sp. X para un uso }}{\text { Núm. total de menciones de todas las spp. para un uso }} * 100 \\
V u t_{z}=\sum\left(V u_{x}+V u_{y}+V u_{z}+\ldots . . .+V u_{n}\right)
\end{gathered}
$$

$I u_{z}$ : Intensidad de uso; $\mathrm{Fm}_{z}$ : Frecuencia de mención; $V u_{z}$ : Valor de uso; $V u t_{z}$ : Valor de uso total para cada especie.

Los resultados y tendencias que se obtuvieron y registraron en este estudio se basaron únicamente en frecuencias y porcentajes. La abundancia de las especies se identificó de la misma manera, ya que el formato de encuesta consideró una sola opción para selección de la especie más abundante, de acuerdo con el conocimiento del área y de la especie.

Con la finalidad de discutir sobre el manejo y conservación de la fauna silvestre, considerando las necesidades de la gente, se utilizó la información acerca del conocimiento y percepción que tienen del jaguar los pobladores de la zona, de la abundancia de las presas y de los factores que afectan su distribución y conservación.

\section{Resultados}

Para el primer propósito se realizaron 42 encuestas. El 79\% las respondieron hombres entre los 17 y los 89 años; de ellos, el 91\% ( $\mathrm{n}=33)$ se dedican a las actividades agropecuarias (siembra de caña y maíz, y cuidado del ganado). El 21\% fueron respondidas por mujeres entre los 22 y 84 años; el $100 \%$ de las mujeres se dedican a las labores de la casa y ayudan con el trabajo de campo.

Los pobladores de San Nicolás de los Montes reconocieron como principales presas silvestres del jaguar a $O$. virginianus, $M$. temama, $P$. tajacu, $N$. narica, $D$. novemcinctus y $C$. paca. Además, reconocieron en menor proporción al conejo (Sylvilagus sp.), zorra (Urocyon cinereoargenteus), coyote (Canis latrans) y algunas aves. Sobresale que $71 \%$ de los encuestados reconocieron como otras presas a especies domésticas (vacas, becerros, caballos, potrillos, mulas, chivos, borregos y perros).

De acuerdo con la percepción de los pobladores, las presas más abundantes, por ser especies con las que se encuentran frecuentemente en el bosque, en cultivos o en caminos, son $P$. tajacu, $N$. narica y $D$. novemcinctus. La mayoría de los encuestados ha observado una disminución en la abundancia de esta fauna a través del tiempo (Fig. 1). Las causas reconocidas por los pobladores de dicha disminución son el aumento en la población humana, la cacería que se realiza y los depredadores naturales (jaguar y puma).

La mayoría de los encuestados considera que los desmontes no afectan la fauna (Fig. 2), por el contrario, la benefician al alimentarse de los cultivos (por ejemplo, $O$. viriginianus, $P$. tajacu y $N$. narica se alimentan del maíz y del frijol) y porque existen suficientes áreas de refugio para la misma. Otros reconocen que los desmontes afectan la fauna silvestre, ya que ésta se aleja y busca protección en áreas más densas; asimismo, creen que el uso de pesticidas en el área agrícola provoca la intoxicación de animales, y un porcentaje menor de los encuestados no sabe si los desmontes la afecta (Fig. 2).

Una parte significativa de los encuestados (89\%) considera que el jaguar al alimentarse de animales domésticos perjudica a las personas. El 11\% menciona que no le hace daño a la gente $y$, aunque le temen, nunca han sido atacados. Los ejidatarios reconocen una disminución de las poblaciones de mamíferos silvestres, pero no la relacionan con la alimentación del jaguar ni con los ataques del felino a los animales domésticos.

Para identificar el conocimiento, uso y valor que los ejidatarios otorgan a estas presas, se realizaron 62 


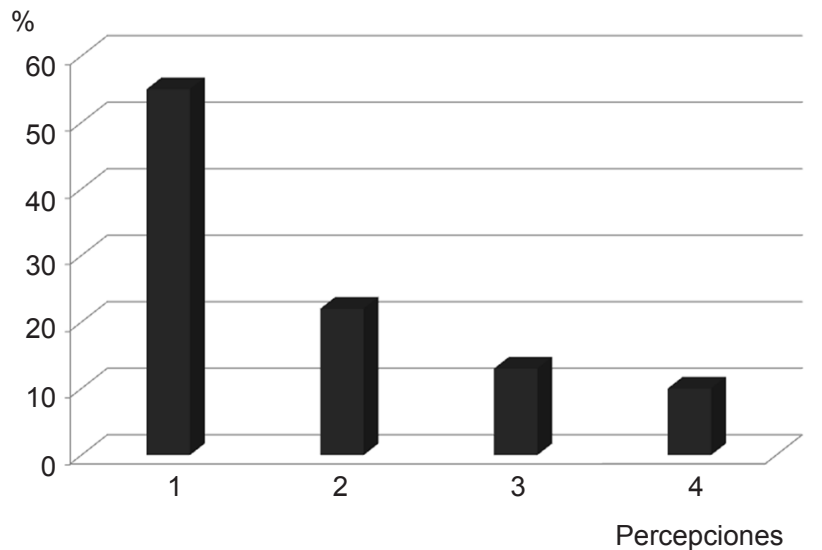

Figura 1. Percepción de los pobladores rurales sobre la abundancia de la fauna silvestre. 1, la abundancia de la fauna silvestre ha disminuido; 2, la abundancia se mantiene; 3, la abundancia es mayor ahora; 4 , no respondieron a esta pregunta.

encuestas, de las cuales el $71 \%$ se dirigieron a hombres entre 17 y 89 años de edad y el $29 \%$ restante a mujeres en edades de 19 a 80 años.

Como principales valores para las 6 especies de presas, los pobladores reconocen los usos alimenticio, ornamental, medicinal, artesanal y religioso; asimismo, reconocen su valor como trofeos de caza deportiva, mascotas y vestido (Fig. 3). La mayoría de los encuestados (82\%) dijo consumir alguna de estas especies y reconocer, por ejemplo, el sabor y el aporte de biomasa de la carne de venado. Los ejidatarios emplean diferentes partes del cuerpo de las especies (Cuadro 1). A continuación se describen los usos que los pobladores de San Nicolás de los Montes mencionaron para a las 6 presas del jaguar y sus productos.

Alimenticio. Odocoileus virginianus, M. temama y $P$. tajacu son las presas que con más frecuencia se consumen; son favoritas por el sabor de su carne y por el aporte de biomasa. También se consumen, aunque no muy frecuentemente, $N$. narica, D. novemcinctus y $C$. paca; los ejidatarios suelen prepararlas en mole, chicharrones o barbacoa.

Medicinal. La sangre de $O$. virginianus cura males cardiacos, y el hígado de esta especie y el de M. temama lo utilizan para combatir la anemia; las astas molidas se utilizan para curar males del oído; la médula de $M$. temama se emplea para controlar los dolores de muelas; la grasa de $N$. narica la usan para eliminar granos e infecciones de la piel, para dolores musculares y para curar la epilepsia. Para aliviar la tos se utiliza la concha de $D$. novemcinctus y su grasa también sirve para curar infecciones de la piel.

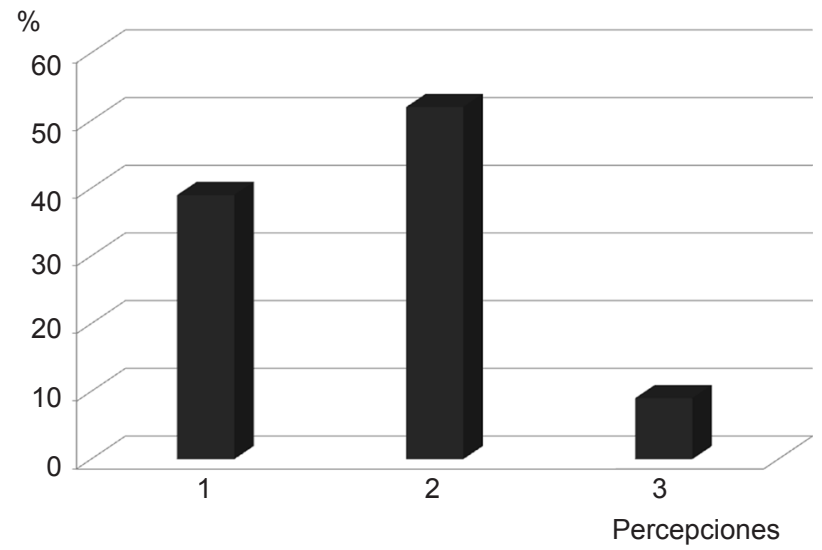

Figura 2. Causas de la disminución de la fauna silvestre. 1, los desmontes afectan la fauna silvestre; 2 , los desmontes no afectan la fauna silvestre; 3 , no saben si los desmontes afectan dicha fauna.

Ornamental. La piel de C. paca se usa como ornato en las casas; de igual forma, los cráneos de venados se utilizan como adornos o percheros.

Vestido. En este ejido las pieles de los animales se utilizan poco para este fin; sin embargo, reconocen que la piel de $O$. virginianus sirve para fabricar botas y chamarras.

Artesanal. De O. virginianus se utilizan las astas para elaborar percheros y pizcadores, la piel para adornar cachas de cuchillos y las patas para hacer cuartas; de M. temama se aprovecha la piel para chaparreras; de P. tajacu se utilizan los colmillos para fabricar collares y de $D$. novemcinctus reconocen que se pueden elaborar instrumentos musicales, aunque esta actividad no la realizan en este ejido.

Los ungulados obtuvieron los valores más altos del índice de importancia cultural (Cuadro 2), debido al sabor de su carne y al uso de su piel y astas, entre otros.

\section{Discusión}

Las principales presas silvestres del jaguar en el ejido San Nicolás de los Montes fueron $O$. virginianus, $M$. temama, P. tajacu, N. narica, D. novemcinctus y C. paca, especies también registradas como tales en otros estudios (Núñez et al., 2000; Kuroiwa y Ascorra, 2006; Weckel et al., 2006). Estas especies han sido identificadas como las de mayor uso alimenticio entre los pobladores del ejido, lo cual los convierte regionalmente en competidores del jaguar (Aranda, 1999; Quijano-Hernández y Calmé, 2002; Treves y Karanth, 2003; Monroy et al., 2008; RosasRosas et al., 2008), conflicto que la población humana mitiga eliminando estos felinos (Weber y Rabinowitz, 1996). Sin embargo, de permanecer esta situación, este 


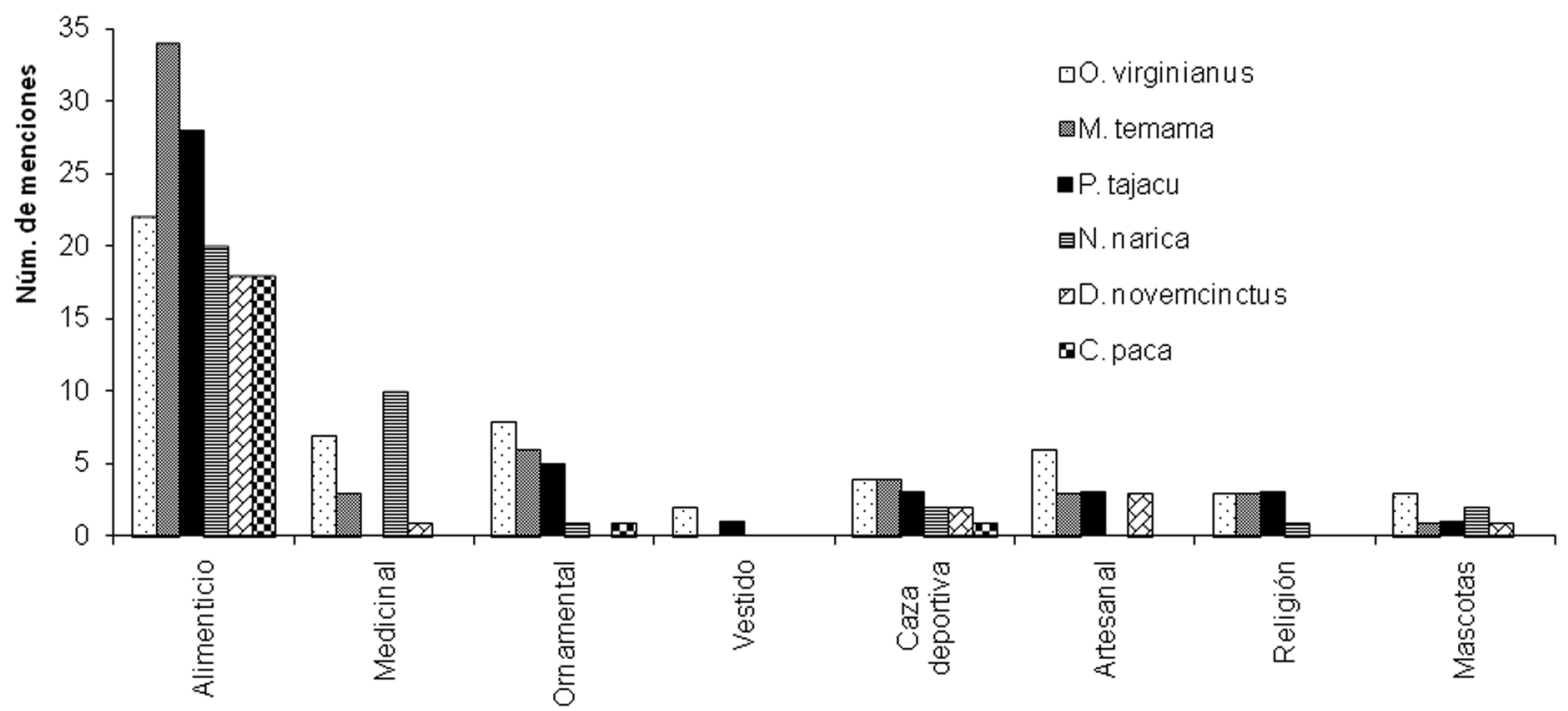

Figura 3. Valores que los pobladores rurales reconocen para las 6 especies.

Cuadro 1. Usos que los pobladores dan a las presas del jaguar y a sus productos en San Nicolás de los Montes, San Luis Potosí, México

\begin{tabular}{|c|c|c|}
\hline Especie & Uso & Parte utilizada \\
\hline Venado cola blanca (O. virginianus) & $\begin{array}{c}\text { Alimenticio, medicinal } \\
\text { ornamental, artesanal } \\
\text { mascota, caza, vestido }\end{array}$ & $\begin{array}{c}\text { Carne, astas, sangre, } \\
\text { Hígado, piel }\end{array}$ \\
\hline Temazate (M. temama) & $\begin{array}{c}\text { Alimenticio, medicinal } \\
\text { ornamental, artesanal } \\
\text { caza }\end{array}$ & Carne, médula \\
\hline Armadillo (D. novemcinctus) & $\begin{array}{c}\text { Alimenticio, medicinal } \\
\text { ornamental, artesanal }\end{array}$ & Carne, grasa, concha \\
\hline Pecarí de collar (P. tajacu) & Alimenticio, artesanal & Carne, colmillos \\
\hline Tejón (N. narica) & Alimenticio, medicinal & Carne, grasa \\
\hline Tuza real (C. paca) & Alimenticio, ornamental & Carne, piel \\
\hline
\end{tabular}

Cuadro 2. Índice de importancia cultural de presas del jaguar en San Nicolás de los Montes, San Luis Potosí, México

\begin{tabular}{lcccccc}
\hline Especie & Núm. de usos & Menciones & Iu & Fm & Vut \\
\hline O. virginianus & 8 & 55 & 22.22 & 23.50 & 35.78 & 27.17 \\
M. temama & 7 & 54 & 19.44 & 23.07 & 19.33 & 20.61 \\
P. tajacu & 7 & 44 & 19.44 & 18.80 & 19.79 & 19.34 \\
N. narica & 6 & 36 & 16.66 & 15.38 & 14.27 & 15.44 \\
D. novemcinctus & 5 & 25 & 13.88 & 10.68 & 7.82 & 10.80 \\
C. paca & 3 & 20 & 8.33 & 8.54 & 2.98 & 6.62 \\
Total & & 234 & 100 & 100 & 100 & 100 \\
\hline
\end{tabular}

$I u=$ Intensidad de uso, $F m=$ Frecuencia de mención, $V u t=$ Valor de uso de las especies, $I I C=$ Índice de importancia cultural. 
tipo de problemas no será erradicado (Distefano, 2004; De Azevedo y Murray, 2007).

De los encuestados sobresale que el $71 \%$ reconocieron como otras presas del jaguar a especies domésticas (vacas, becerros, caballos, potrillos, mulas, chivos, borregos y perros), información citada en otros estudios (Scognamillo et al., 2006; Rosas-Rosas et al., 2008; Cavalcanti y Gese, 2010) y que constituye un conflicto adicional entre ganaderos y jaguares, colocando al jaguar bajo continua amenaza. En Centro y Sur América, los conflictos con jaguares dentro de las áreas ganaderas son comunes y provocan pérdidas económicas y muertes reiteradas de jaguares (Zimmermann et al., 2005) un fenómeno similar ocurre en algunas regiones del norte de México (Rosas-Rosas et al., 2008).

Se ha observado una relación entre la disponibilidad de las presas silvestres y el consumo de animales domésticos por el jaguar; cuando las presas silvestres disminuyen, el consumo de domésticos se incrementa (Cavalcanti y Gese, 2010). Por ello, a medida que las presas silvestres del jaguar sean utilizadas por los pobladores y las actividades agrícolas y pecuarias se incrementen en las zonas rurales, los conflictos entre ganaderos y jaguares persistirán (Rosas-Rosas et al., 2008).

La degradación del hábitat, los cambios en el uso de suelo, la contaminación y la explotación ilegal de los recursos naturales, son acciones que colocan al jaguar y a sus presas en una presión constante (Sánchez et al., 2002; Valdez et al., 2009). En el área de estudio se evidenciaron cambios en el uso del suelo para la producción de maíz y caña de azúcar. Leyequién y Balvanera (2007) informaron la pérdida de aproximadamente 10000 ha de áreas naturales transformadas en zonas de cultivo en el Carso Huasteco. A medida que las áreas naturales se deforestan se afecta la supervivencia del jaguar y de sus presas, situación que constantemente se agrava dado el requerimiento espacial tan amplio de este felino (Leite y Franklin, 2002). Adicionalmente, Aldana et al. (2006) mencionan que el uso indiscriminado de agroquímicos afecta a la fauna silvestre, actividad que ocurre en la región y que de no controlarse, podría tornarse perjudicial cuando la fauna silvestre consuma estos cultivos. Los pobladores percibieron una disminución en la abundancia de las especies de fauna silvestre que atribuyen a la pérdida de hábitat, al cambio en el uso de suelo, a la fragmentación y a la caza de algunas especies, como lo han registrado en otras regiones Mosa y Goitia (2004). La cacería que se realiza en la zona para autoconsumo o para comercialización afecta el tamaño de las poblaciones silvestres, principalmente las de mamíferos medianos y grandes (Ojasti, 2000).

Los pobladores de San Nicolás de los Montes, al igual que en otras comunidades rurales de América, tienen conocimiento acerca de las especies de fauna silvestre y sus usos (Guerra-Roa et al., 2004). Ojasti (2000) menciona que los usos que los pobladores otorgan a la fauna silvestre varían de región a región y están ligados principalmente a sus costumbres. Los usos específicos que los ejidatarios de SNM le otorgan a la fauna silvestre son el alimenticio, ornamental, medicinal, artesanal, caza deportiva, religión, mascotas y vestido. Aldana et al. (2006) identificaron en Colombia 14 usos, siendo el de la cacería el más importante, el cual conlleva a otros usos como el artesanal, comercial y medicinal. En general, la cacería de especies silvestres es el uso más importante registrado en México y en Latinoamérica (Mellink et al., 1986; Aranda et al., 1999; Ojasti, 2000; Tejada et al., 2006; Monroy -Vilchis et al., 2008). En San Nicolás de los Montes, las especies que presentaron mayor valor de uso e importancia cultural fueron $O$. viriginianus (27.17), M. temama (20.61) у $P$. tajacu (19.34), especies que coinciden con lo registrado en Latinoamérica por Ojasti (2000).

El presente estudio demostró una demanda del ejido por la fauna silvestre, particularmente de aquellas especies que son presas naturales del jaguar. Es muy probable que ante una situación de ausencia o disminución significativa de las presas del jaguar en la región, se presenten ataques de felinos a animales domésticos (Hoogesteijn y Hoogesteijn, 2005) y, mientras el hombre y animales silvestres compitan por los mismos recursos, el conflicto entre los humanos y la vida silvestre podrían persistir (Distefano, 2004; De Azevedo y Murria, 2007), ocasionando pérdidas económicas y muertes de jaguares (Zimmermann et al., 2005).

El reto es lograr que los pobladores rurales y las presas del jaguar persistan en el área de estudio bajo condiciones apropiadas y a largo plazo, y que los ataques por este felino a los animales domésticos disminuya en el corto plazo. Para lograr este propósito, es importante entender las causas reales que motivan que un jaguar ataque y consuma animales domésticos. Mcdonald y Sillero-Zubiri (2002) mencionan que estos ataques están relacionados con las características del paisaje, distancia a asentamientos humanos, con la disponibilidad de cobertura, de la distribución de sus presas naturales y con las características generales del hábitat. Sáenz y Carrillo (2002) han sugerido que la presencia del jaguar en zonas de pastoreo de domésticos, se debe a una reducción de sus presas naturales a causa de la cacería, a la destrucción del hábitat y a la dispersión de individuos jóvenes que por falta de territorios en las áreas naturales, se establecen en los potreros (Sáenz y Carrillo, 2002). Michalski et al. (2006) particularmente relacionan este conflicto con la proximidad del ganado a las zonas arboladas y ribereñas, con los picos reproductivos de las especies domésticas, las estaciones 
del año y con la cantidad de ganado en los potreros. En el ejido los pobladores indicaron una disminución en la abundancia de presas para el jaguar y reportaron ataques del felino a animales domésticos. Es posible que éstos sean el reflejo de una disminución de sus presas silvestres por afectaciones al hábitat, a un incremento en la cacería de subsistencia o a un mal manejo del ganado en el área.

Para lograr que los pobladores rurales y las presas del jaguar persistan en el área de estudio en números apropiados, y que los ataques por este felino a los animales domésticos, disminuya con el tiempo, es necesario diseñar una estrategia de manejo y conservación integral de los recursos naturales incluyendo el diseño de mejores prácticas en el manejo de especies domésticas en el área.

El primer paso de la estrategia es realizar un diagnóstico de los recursos naturales con que cuenta el ejido, que incluya mayor conocimiento de éstos y de su estado; llevar a cabo una zonificación del área de acuerdo con el grado de conservación de sus hábitats; definir la distribución de potreros; conocer las características de la vegetación y del paisaje, y de las condiciones del hábitat en cada uno; conocer la distribución de aguajes naturales y artificiales, entre otros. En este proceso, es muy importante organizar a los pobladores rurales, educarlos ambientalmente (aconsejándolos de que la fauna silvestre bien manejada, incluyendo al jaguar, constituye para ellos una oportunidad de ingreso económico complementario) e involucrarlos en las tareas para que obtengan un mayor conocimiento de sus recursos naturales y un mejor sentido de su manejo y conservación.

Un segundo paso es asesorar al ejido San Nicolás de los Montes para que se constituya como una Unidad de Manejo para la Conservación de Vida Silvestre en sus diversas modalidades y capacitar a los ejidatarios en el manejo apropiado de su ganado, a fin de que que reciban pagos por Servicios Ambientales.

El tercer paso sería, de ser el caso 1), conducir acciones para mejorar el hábitat en sitios muy específicos y de esa manera incrementar las presas del jaguar, ya que este felino prefiere el consumo de presas silvestres (Patterson et al., 2004; Odden et al., 2008) y 2), manejar adecuadamente las especies domésticas.

Son varias las acciones que sobre mejoramiento de hábitat y manejo de animales domésticos se han propuesto para mitigar problemas relacionados con el jaguar (Sunquist y Sunquist, 1989; Aranda, 1994; Nuñez et al., 2002). Sin embargo, sobresalen la construcción y uso de aguajes durante la estación seca y, en casos extremos, la reintroducción de especies presa (Linnell et al., 1999; Patterson et al., 2004; Palmeira et al., 2008). Con respecto al manejo del ganado, Ogada et al. (2003) recomiendan evitar nacimientos de ganado en las proximidades de las zonas boscosas. El presente estudio constituye la base para la realización de futuros trabajos específicos tendientes a manejar y conservar los recursos naturales del ejido San Nicolás de los Montes de una manera más integral y así lograr un desarrollo rural sustentable en beneficio de sus habitantes.

\section{Agradecimientos}

Al Consejo Nacional de Ciencia y Tecnología (CONACYT), a la Secretaría de Desarrollo Agropecuario y Recursos Hidráulicos (SEDARH) del Estado de San Luis Potosí, a la comunidad de San Nicolás de los Montes, especialmente a las familias Villalón y Moctezuma, al Dr. José G. Herrera Haro y al Dr. Raúl Valdez por la revisión del documento.

\section{Literatura citada}

Aldana, N., M. Díaz, A. Feijoo y M. C. Zúñiga. 2006. Valoración del uso de la fauna silvestre en el Municipio de Alcalá, Valle del Cauca. Scientia et Technica 12:291-297.

Aranda, M. 1994. Importancia de los pecaríes (Tayassu spp.) en la alimentación del jaguar (Panthera onca). Acta Zoológica Mexicana (n.s.) 62:11-22.

Aranda M., M. Gual-Díaz, O. Monrroy-Vilchis, L. Silva y A. Velázquez. 1999. Aspectos etnoecológicos: aprovechamiento de la flora y fauna silvestre en el sur de la Cuenca de México. In Biodiversidad de la región de montaña del sur de la cuenca de México, A. Velázquez y F. Romero (eds.). Universidad Autónoma Metropolitana Secretaría del Medio Ambiente, México, D. F. p. 263- 275.

Barbarán, R. R. 2004. Usos mágicos, medicinales y rituales de la fauna en la puna del noroeste Argentino y sur de Bolivia. Contribuciones al Manejo de Vida Silvestre en Latino América 1:1-26.

Bezaury-Creel, J. y D. Gutiérrez Carbonell 2009. Áreas naturales protegidas y desarrollo social en México. Conservación de la biodiversidad en México. In Capital natural de México (J. Sarukhán (coord. general). Comisión Nacional para el Conocimiento y Uso de la Biodiversidad (CONABIO), México, D. F. p. 385-431.

Cavalcanti, S. y E. M. Gese. 2010. Kill rates and predation patterns of jaguar (Panthera onca) in the southern Pantanal, Brazil. Journal of Mammalogy 91:722-736.

Ceballos, G., J. Arroyo-Cabrales y R. A. Medellín. 2002. The mammals of México: composition, distribution, and status. Occasional papers, Texas Tech. University 218:1-27.

Ceballos G. y G. Oliva. 2005. Los Mamíferos silvestres de México. Fondo de Cultura Económica/ Comisión Nacional para el Conocimiento y Uso de la Biodiversidad, México, D. F. 981 p. 
Challenger, A. 1998. Utilización y conservación de los ecosistemas terrestres de México. Pasado, presente y futuro. CONABIO/ UNAM/ SIERRA MADRE, México, D. F. 847 p.

CONABIO (Comisión Nacional para el Conocimiento y Uso de la Biodiversidad). 2000. Estrategia Nacional sobre la Biodiversidad de México. Cultura Ecológica, México, D. F. $76 \mathrm{p}$.

Davies-Mostert, H., C. Hodkinson, H. Komen y T. Snow. 2007. Predators and farmers. Endagered Wildlife Trust, Johannesburg. $30 \mathrm{p}$.

De Azevedo F. C. y D. Murray. 2007. Evaluation of potential factors predisposing livestock to predation by jaguars. The Journal of Wildlife Management 71:2379-2386.

Distefano, E. 2004. Human-Wildlife Conflict worldwide: collection of case studies, analysis of management strategies and good practice. SAED/ FAO, Roma. 34 p.

Figueroa-Solano, E. 2000. Uso agroecológico, actual y potencial, de especies arbóreas en una selva baja caducifolia perturbada del suroeste del Estado de México. Tesis, Maestría Colegio de Postgraduados, Montecillo, Texcoco, Estado de México. 38-39 p.

Guerra-Roa, M., E. Naranjo, F. Limón y R. Mariaca y Méndez. 2004. Factores que intervienen en la regulación local de subsistencia en dos comunidades de la selva lacandona, Chiapas. In Memorias, Manejo de Fauna Silvestre en la Amazonia y Latinoamérica. Universidad Nacional de la Amazonia Peruana, Iquito, Loreto 540-550 p.

Hoogesteijn R. y A. Hoogesteijn. 2005. Manual sobre los problemas de depredación causado por jaguares en ganado de corte, Wildlife Conservation Society, New York. 46 p.

Hoogesteijn, R., E. Boede y E. Mondolfi. 2006. Observaciones de la depredación de bovinos por jaguares en Venezuela y los programas gubernamentales de control. In El jaguar en el nuevo milenio, R. Medellín, A. Equihua, C. Chetkiewics, A. Rabinowitz, P. Crawshaw, A. Rabinowitz, K. Redford, J. Robinson, E. Sanderson y A. Taber (eds.). Fondo de Cultura Económica/ Universidad Nacional Autónoma de México/ Wildlife Conservation Society, México, D. F. p. 183-197.

INEGI (Instituto Nacional de Estadística Geografía e Informática). 2003. Síntesis de información geográfica del estado de San Luis Potosí. Aguascalientes, Aguascalientes. Versión digital. http://www.inegi.org.mx/sistemas/productos/default.aspx?c $=265 \&$ s $=$ inegi\&upc $=702825224356 \& p f=$ Prod\&ef $=\& f=1 \& c$ $1=0 \& \operatorname{tg}=43 \& p g=0 \#$ inicio; última consulta: $8 . X .2007$.

INEGI (Instituto Nacional de Estadística Geografía e Informática). 2008. Principales Resultados por Localidad 2005 (ITER), como parte del Programa de Divulgación de Resultados del II Conteo de Población y Vivienda 2005. Instituto Nacional de Estadística Geografía e Informática, Aguascalientes, Aguascalientes. www.INEGI.org.mx/est/ contenidos/espanol/sistemas/conteo2005/ITER2005/default. aspx; última consulta:10.VII.2009.
Kuroiwa, A. y C. Ascorra. 2006. Dieta y densidad de posibles presas de jaguar en las inmediaciones de la zona de reserva Rambopata-Candamo, Perú. In El jaguar en el nuevo milenio, R. Medellín, A. Equihua, C. Chetkiewics, A. Rabinowitz, P. Crawshaw, A. Rabinowitz, K. Redford, J. Robinson, E. Sanderson y A. Taber (eds.). Fondo de Cultura Económica/ Universidad Nacional Autónoma de México/ Wildlife Conservation Society, México, D. F. p. 199-207.

Leite R. y G. Franklin. 2002. El jaguar, el puma y el hombre en tres áreas protegidas del bosque atlántico costero de Paraná, Brasil. In El jaguar en el nuevo milenio, R. Medellin, C. Equihua, C. Chetkiewicz, P. Crawshaw, A. Rabinowitz, A. Redford, J. Robinson, E. Sanderson y A. Taber (eds.). Fondo de Cultura Económica, Universidad Autónoma de México, Wildlife Conservation Society. p. 237-250.

Leyequién, L. y R. M. Balvanera. 2007. El jaguar en el este de la Huasteca potosina. In Conservación y manejo del jaguar en México. Estudio de caso y perspectivas, G. Ceballos, C. Chávez, R. List y H. Zarza (eds.). CONABIO/ WWF/ Telcel/ UNAM, México, D. F. p. 51-58.

Linnell J., J. Odden, M. Smith, R. Aanes y J. Swenson. 1999. Large carnivores that kill livestock: do "problem individuals" really exist? Wildlife biology 27:698-705.

López-Cabrera, C., X. Zazueta-Angulo y C. Porras-Andujo. 2005. Notas sobre aprovechamiento del medio ambiente norestense: reconocimiento del terreno, inicio de la cacería. Ra Ximhai 1:39-52.

Michalski, F., R. Boulhosa, A. Faria y C. Peres. 2006. Humanwildlife conflicts in a fragmented amazonian forest landscape: determinants of large felid depredation on livestock. Animal Conservation 9:179-188.

Mellink, E., J. R. Aguirre y J. M. García. 1986. Utilización de la fauna silvestre en el altiplano potosino-zacatecano. Centro Regional para Estudios de Zonas Áridas y Semiaridas, Colegio de Postgraduados, Campus San Luis Potosí. 104 p.

Monroy-Vilchis, O., L. Cabrera, P. Suárez, M. Zarco-González, C. Rodríguez-Soto y V. Urios. 2008. Uso tradicional de vertebrados silvestres en la sierra de Nanchititla, México. Interciencia 33:308-313.

Mosa, S. y M. Gotia. 2004. Evaluación de la caza recreativa sobre la fauna silvestre en las provincias de Salta y Jujuy, Argentina. In Memorias, Manejo de Fauna Silvestre en la Amazonia y Latinoamérica. Universidad Nacional de la Amazonia Peruana, Iquitos, Loreto. p. 40-550.

Núñez R., B. Miller y F. Lindsey. 2002. Ecología del jaguar en la reserva de la Biosfera Chamela-Cuixmala, Jalisco, México. In $\mathrm{El}$ jaguar en el nuevo milenio, R. Medellin, C. Equihua, C. Chetkiewicz, P. Crawshaw, A. Rabinowitz, A. Redford, J. Robinson, E. Sanderson y A. Taber (eds.). Fondo de Cultura Económica, Universidad Autónoma de México, Wildlife Conservation Society, México, D. F. p. 107-126.

Núñez, R., B. Miller y F. Lindzey. 2000. Food habits of jaguar and 
pumas in Jalisco, México. Journal of Zoology 252:373-379.

Odden, J., I. Herfindal, J. Linnell y R. Andersen. 2008. Vulnerability of domestic sheep to lynx depredation in relation to roe deer density. Journal of Wildlife Management 72:276-282.

Ogada, M., R. Woodroffe, N. Oguge y L. Frank. 2003. Limiting depredation by African carnivores: the role of livestock husbandry. Conservation Biology 17:1521-1530.

Ojasti, J. 2000. Manejo de fauna neotropical. Instituto de Zoología Tropical, Venezuela. 304 p.

Palmeira, F., P. Crawshaw, C. Haddad, K. Ferraz y L. Verdade. 2008. Cattle depredation by puma (Puma concolor) and jaguar (Panthera onca) in centralwestern. Brazil. Biological Conservation 141:118-125.

Patterson, B., S. Kasiki, E. Selempo y R. Kays. 2004. Livestock predation by lions (Panthera leo) and others carnivores on ranches neighboring Tsavo National Parks, Kenya. Biological Conservation 119:507-516.

Puig, H. 1991. Vegetación de la Huasteca, México. Estudio fitogeográfico y ecológico. Institut Francais de Recherche Scientifique pour le Développement en Coopération (ORSTOM)/ Instituto de Ecología/ Centre D' Études Mexicaines et Centramericaines (CEMCA), México, D. F. $632 \mathrm{p}$.

Purdy, K. y D. Decker. 1989. Applying wildlife values information in management: the wildlife attitudes and values scale. Wildlife Society Bulletin 17:494-500.

Quijano-Hernández, E. y S. Calmé. 2002. Patrones de cacería y conservación de la fauna silvestre en una comunidad maya de Quintana Roo, México. Etnobiología 2:1-18.

Ramamoorthy, T. P., R. Bye., A. Lot y J. Fa. 1998. La diversidad biológica de México. Orígenes y distribución. Instituto de Biología, UNAM, México, D. F. 739-757 p.

Rosas-Rosas, O., L. Bender y R. Valdez. 2008. Jaguar and puma predation on cattle calves in northeastern Sonora, Mexico. Rageland Ecology and Management 61:554-560.

Sáenz, J. y E. Carrillo. 2002. Jaguares depredadores de ganado en Costa Rica: ¿un problema sin solución? In El jaguar en el nuevo milenio, R. Medellín, C. Equihua, C. Chetkiewicz, P. Crawshaw, A. Rabinowitz, A. Redford, J. Robinson, E. Sanderson y A. Taber (eds.). Fondo de Cultura Económica/ Universidad Nacional Autónoma de México/ Wildlife Conservation Society, México D. F. p. 127-138.
Sánchez O., J. Ramírez- Pulido, U. Aguilera-Reyes y O. MonroyVilchis. 2002. Felid record from the State of México, México. Mammalia 66:289-294.

Scognamillo, D. I., E. Maxit, M. Sunquist y L. Farell. 2006. Ecología del jaguar y el problema de la depredación de ganado en un hato de los llanos Venezolanos. In El jaguar en el nuevo milenio, R. Medellín, C. Equihua, C. Chetkiewicz, P. Crawshaw, A. Rabinowitz, A. Redford, J. Robinson, E. Sanderson y A. Taber (eds.). Fondo de Cultura Económica/ Universidad Nacional Autónoma de México/ Wildlife Conservation Society, México, D. F. p. 139-149.

Sunquist, M. E. y F. Sunquist. 1989. Ecological constraints on predation by large felids. In Carnivore behavior, ecology and evolution, J. L. Gittleman (ed.). Cornell University Press, New York. p. 283-301.

Tejada, R., E. Chao, H. Gómez, E. Painter y R. Wallace. 2006. Evaluación sobre el uso de la fauna silvestre en la tierra comunitaria de origen, Tacana, Bolivia. Ecología en Bolivia 41:138-148.

Toledo, V. M. 1988. La diversidad biológica de México. Ciencia y Desarrollo 81:17-30.

Treves, A. y U. Karanth. 2003. Human-carnivore conflict and perspectives on carnivore managment worldwide. Conservation Biology 17:491-1499.

Turner, N. 1988. The importance of a rose. Evaluating the cultural significance of plants in Thompson and Lilloet Interior Salish. American Anthropologist, new series 90:272-290.

Valdez, R., J. C. Guzmán-Aranda, F. J. Aberca y L. TarangoArámbula. 2006. Wildlife conservation and management in Mexico. Wildlife Society Bulletin 34:270-282.

Villordo, G. A. 2009. Distribución y estado de conservación del jaguar (Panthera onca) en San Luis Potosí, México. Tesis, Maestría, Colegio de Postgraduados, Montecillo, Estado de México, México, D. F. 84 p.

Weber, W. y A. Rabinowitz. 1996. A global perspective on large carnivore conservation. Conservation Biology 10:1046-1054.

Weckel, M., W. Giuliano y S. Silver. 2006. Jaguar (Panthera onca) feeding ecology: distribution of predator and prey through time and space. Journal of Zoology: doi:10.1111/ j.1469-7998.2006.00106.x.

Zimmermann, A., M. Walpole y N. Leader-Williams. 2005. Cattle ranchers' attitudes to conflicts with jaguar Panthera onca in the Pantanal of Brazil. Oryx 39:406-412. 